\title{
Small intestinal mucosal protection mechanisms and their importance in rheumatology
}

\author{
Seamus O’Mahony, Anne Ferguson
}

Disturbances in intestinal immunity and other protective mechanisms may have a pivotal role in the cause of certain inflammatory joint diseases. The association between inflammatory bowel disease and arthritis is well established, ${ }^{1}$ as is the association between intestinal infection with organisms such as yersinia and a reactive seronegative arthritis. Whipple's disease and jejunoileal bypass surgery ${ }^{2}$ may result in a spondylitis or a reactive arthropathy. These well established associations have prompted research into the relation between disturbances in small intestinal function and inflammatory joint diseases. For example, many patients with ankylosing spondylitis have inflammatory lation has centred on the putative association between this condition and intestinal carriage of Klebsiella pneumoniae. ${ }^{4}$ Patients with rheumatoid arthritis and ankylosing spondylitis may have disturbances of small intestinal mucosal permeability, independent of the effect of nonsteroidal anti-inflammatory drugs (NSAIDs). ${ }^{5}$ Treatment of inflammatory joint diseases may adversely effect the small intestine. Much interest has focused on the effects of NSAIDs on the small intestine, in particular, their effects on small intestinal mucosal permeability. ${ }^{6}$ Bjarnason et al have shown that these agents produce inflammatory changes of the small intestine in a significant percentage of patients. ${ }^{7}$

The notion of an intestinal cause of inflammatory joint diseases is not new. In the 1920 s colectomies were performed as a therapeutic measure in patients with rheumatoid arthritis, and Svartz (who first described the treatment of rheumatoid arthritis with sulphasalazine) proposed an enteric cause of this condition in the 1940 s. $^{8}$

The first part of this review is concerned with immune defences of the small intestine and disturbance of these defences in rheumatic conditions; the second part deals with the intestinal epithelial barrier, mucosal permeability of the small intestine, and disturbances of intestinal permeability in rheumatic conditions.

Gastrointestinal Unit, Western General Hospital and University of Edinburgh, of Edinburgh, Erewe Road, 4 2XU S O'Mahony A Ferguson

Correspondence to Dr O'Mahony.

Intestinal immunity and inflammatory joint disease

The mucosal surfaces of the body, such as those of the gastrointestinal and respiratory tracts, provide effective barriers to the entry of potentially harmful substances and infectious agents. lesions of the terminal ileum, ${ }^{3}$ and much specu-

Immune and non-immune mechanisms operate to prevent colonisation and invasion by the vast array of potential pathogens to which the secretory mucosae are constantly exposed. In the gastrointestinal tract non-immune mechanisms of defence include gastric acid, normal bowel flora, intraluminal proteases, and goblet cell mucus. Intestinal epithelial cells efficiently exclude macromolecules while allowing absorption of nutrients, water, and electrolytes. The most important agents of the immune mechanisms are the secretory immunoglobulins and antibodies found in the external secretions bathing mucosal surfaces. These antibodies inhibit bacterial adherence, neutralise viruses and toxins, and prevent the absorption of potentially harmful antigens.

PHYSIOLOGY OF INTESTINAL HUMORAL IMMUNITY The intestine is the most important immunoglobulin and antibody forming organ of the human body. There are about $10^{10}$ immunoglobulin producing immunocytes per metre of small intestine, $70-90 \%$ of which produce $\operatorname{IgA}$ only. ${ }^{9}$ Intestinal immunocytes produce mainly secretory IgA, a dimer linked by a polypeptide called the 'joining' or $\mathrm{J}$ chain. ${ }^{10}$ (Serum IgA is mainly monomeric.) The IgA is transported through the glandular epithelium by a receptor produced by the epithelium called secretory component. ${ }^{11}$ Secretory component interacts with a specific binding site on the Fc region of the IgA molecule to stabilise its quaternary structure, thus increasing its resistance to proteolytic degradation. ${ }^{12}$ It has been estimated that the amount of secretory $\operatorname{IgA}$ secreted into one metre of small intestine is $0.8 \mathrm{~g} /$ day. ${ }^{13}$

The functional properties of secretory IgA reflect its antigen binding properties. Secretory IgA protects against a variety of foreign antigens, including food antigens, bacteria, viruses, and toxins. It can neutralise viruses and toxins and prevent the adherence of bacterial pathogens to epithelial surfaces. ${ }^{14}$ The effectiveness of secretory IgA as a neutralising antibody against viruses is shown by the responses to the Sabin oral live poliovirus vaccine where protection correlates with concentrations of secretory antisites, allowing it to function effectively as an agglutinin. Unlike IgM and IgG, however, secretory IgA lacks potent effector functions such as classical complement activation, ${ }^{16}$ and thus is not efficient in killing micro-organisms. 
This lack of complement activation may serve a protective function: the vulnerable gut mucosa normally shows no inflammation and virtually no IgG response despite the influx of small amounts of intact dietary antigens following meals. ${ }^{17}$ Antigen uptake is, however, markedly increased in intestinal inflammation and after disruption of the epithelial barrier by NSAID induced damage. ${ }^{18}$

Induction of the secretory immune response is dependent on the source of antigen: whereas an orally encountered antigen stimulates a prompt and longlasting mucosal immune response, parenteral immunisation does not generally induce a secretory $\operatorname{IgA}$ response. ${ }^{19}$ Peyer's patches and intestinal lymphoid follicles have a central role in the uptake and processing of orally encountered antigens before initiating an immune response. Peyer's patches have a specialised epithelium that facilitates uptake and presentation of luminal antigens. ${ }^{20}$ The epithelium contains the specialised $M$ cells (membranous or microfold); these cells take up luminal antigens by endocytosis and transport them to adjacent macrophages and lymphocytes, which are enveloped by $\boldsymbol{M}$ cell cytoplasm. $M$ cells transport a variety of luminal macromolecules, as well as intact viruses, bacteria, and parasites. ${ }^{21}$ Antigens are processed and presented to $T$ helper cells by macrophages and dendritic cells. IgA lymphoblasts are induced by regulatory $T$ cells, including $T$ cells specific for IgA, and 'switch' cells, which change B lymphocytes from $\operatorname{IgM}$ to $\operatorname{IgA}$ production. After stimulation with antigen within the Peyer's patches ${ }^{22}$ lymphoblasts migrate to the draining mesenteric lymph nodes and then through the thoracic duct to the general circulation before returning to the mucosal surfaces. During migration lymphocytes undergo further clonal expansion and differentiation before migrating back to the intestinal lamina propria and other mucosal sites, such as the lachrymal, salivary, and mammary glands. The fact that antigenic priming at gut level gives rise to a secretory immune response not only in the gut, but also in other mucosal surfaces, forms the basis of the concept of the 'common mucosal immune system'.23 For example, oral immunisation with cholera vaccine gives rise to a specific secretory IgA not only in the gut, but also in the mammary, lachrymal, and salivary glands. ${ }^{24}$

POSSIBLE INTESTINAL IMMUNOPATHOGENIC MECHANISMS IN THE CAUSE OF INFLAMMATORY ARTHRITIDES

Defects in the intestinal mucosal barrier or failure of immune exclusion may lead to increased absorption of potentially toxic luminal antigens. Covalent complexes of peptidoglycan and polysaccharide, the primary structural component of bacterial cell walls, have been shown to induce inflammatory arthritis in laboratory animals after local or systemic injection. ${ }^{25} \mathrm{High}$ concentrations of serum IgA antibody to peptidoglycan have been reported in experimental small bowel bacterial overgrowth, ${ }^{26}$ and in patients with inflammatory bowel disease (particularly in those patients with extra- intestinal manifestations, such as arthritis). ${ }^{27}$ Patients with rheumatoid arthritis and ankylosing spondylitis also have increased concentrations of serum IgA antibodies to peptidoglycan, ${ }^{28}$ suggesting increased absorption of bacterial polymers across the intestinal mucosa, resulting in an IgA immune response. Peptidoglycan has been shown to have several proinflammatory effects, such as the activation of macrophages to become cytotoxic and release interleukin-1, complement activation, and stimulation of B lymphocytes. ${ }^{25}$ These several observations support the hypothesis that bacterial cell wall polymers, derived both from commensal organisms and pathogenic agents, may initiate inflammatory arthritis.

These bacterial products must, however, cross the mucosal barrier before initiating the immunopathogenic events described above. Is there any evidence of primary intestinal inflammation in patients with inflammatory arthritis? We have seen that the normal intestinal mucosa usually shows virtually no IgG response, and numbers of intestinal immunocytes secreting IgG are sparse compared with those secreting IgA. It is well reported that patients with inflammatory bowel disease have a disproportionate increase in the number of intestinal plasma cells secreting $\operatorname{IgG},{ }^{29}$ and it has been proposed that such cells may have a central role in the inflammatory process by initiating complement activation. ${ }^{30}$ Much interest has focused on immunocytes in patients with inflammatory arthritis. Patients with ankylosing spondylitis have increased numbers of rectal cells producing IgG, ${ }^{31}$ and elegant ileocolonoscopic studies have shown inflammatory ileocaecal lesions in most of these patients. ${ }^{3}$ These findings were independent of NSAID intake, and these lesions were rare in patients with rheumatoid arthritis. Conversely, Bjarnason et $a l$, using radiolabelled leucocyte scans, reported that two thirds of patients with rheumatoid arthritis had ileocaecal inflammation, and that these changes occurred only in patients treated with NSAIDs. ${ }^{7}$ Segal et al, however, reported that abnormal leucocyte scans in patients with rheumatoid arthritis were independent of NSAID intake. ${ }^{32}$

Are these intestinal inflammatory lesions in arthritic patients of any significance? Rooney $e t$ al reported a patient with rheumatoid arthritis with a positive leucocyte scan, who on endoscopic examination had no evidence of inflammation. ${ }^{33} \mathrm{We}$ have investigated this question in patients with ankylosing spondylitis. We measured concentrations of various proteins in gut lavage fluid (IgG and albumin); concentrations of these proteins reflect disease activity in inflammatory bowel disease (unpublished data). We found no significant increase in concentrations of these gut lavage proteins in a group of 14 patients with ankylosing spondylitis (unpublished observations).

The reported intestinal inflammatory changes may be subclinical, but may be intimately involved in the immunopathogenic process, by allowing ingress of pathogenic bacterial polymers to the intestinal lamina propria. Sulphasalazine is a vital part of the jigsaw: this agent is an 
effective second line treatment for rheumatoid arthritis; possibly its effects are mainly local, acting to diminish intestinal inflammation and altered mucosal permeability. Indeed, Bjarnason et al reported that sulphasalazine significantly decreased small intestinal inflammation in patients with rheumatoid arthritis. ${ }^{34}$ Curiously, there was no correlation between the effects of sulphasalazine on the intestine and the joints. The question of intestinal inflammation in inflammatory arthritis thus remains to be resolved.

\section{ALTERATIONS OF MUCOSAL IMMUNITY IN PATIENTS WITH ARTHRITIS}

Unfortunately, much work in this area has tended to concentrate on serum studies; mucosal immunity is to a great extent independent of systemic immunity, and findings based on serum studies cannot always be extrapolated to represent events at the mucosal level.

Ankylosing spondylitis Patients with ankylosing spondylitis have increased concentrations of serum IgA, ${ }^{35}$ though it is still not entirely clear whether these increased concentrations correlate with disease activity. ${ }^{36}$ The putative association between Klebsiella pneumoniae and this condition has received much attention: it has been proposed that antibodies to this organism cross react with the HLA-B27 histocompatibility antigen. ${ }^{4}$ Patients with active disease have increased rates of faecal carriage of klebsiella organisms, ${ }^{37}$ and several studies have reported high concentrations of serum $\operatorname{IgA}$ antibody to klebsiella in patients with ankylosing spondylitis ${ }^{3839}$; this suggests that an abnormal intestinal immune response to this organism triggers off the condition in genetically susceptible subjects. Other studies, however, failed to report high concentrations of serum IgA antibody to klebsiella, ${ }^{40}{ }^{41}$ and it is not clear whether this immune response is specific to ankylosing spondylitis. Cooper et al, for instance, found high concentrations of IgA antiklebsiella antibody not only in patients with ankylosing spondylitis but also in those with rheumatoid arthritis and inflammatory bowel disease. $^{42}$ Despite the numerous studies on serum IgA anti-klebsiella antibodies, research into the intestinal immune response has been conspicuously absent. With this in mind we examined the intestinal immune response to Klebsiella pneumoniae in ankylosing spondylitis and in inflammatory bowel disease. ${ }^{43}$ We found high concentrations of serum IgA antibody in patients with ankylosing spondylitis (which correlated with the erythrocyte sedimentation rate), but also in patients with inflammatory bowel disease. Intestinal antibody concentrations were similar to those of controls in the patients with ankylosing spondylitis, but markedly raised in the patients with inflammatory bowel disease. It is likely that the mucosal immune response in inflammatory bowel disease is nonspecific, representing ingress to the lamina propria of bacterial antigens across the inflamed mucosa. The ankylosing spondylitis/klebsiella controversy is set to continue.

Rheumatoid arthritis Patients with rheuma- toid arthritis also have increased concentrations of circulating $\operatorname{IgA}$, and $\operatorname{IgA}$ immune complexes correlate with disease activity. ${ }^{44}$ IgA rheumatoid factor is predominantly polymeric $\operatorname{IgAl}^{45}$ and possibly intestinal in origin, as inflamed intestinal mucosa produces increased amounts of $\operatorname{IgAl} .^{46}$ Studies of $\operatorname{IgA}$ in rheumatoid arthritis, however, have tended to be speculative and based on serum findings.

Reiter's syndrome/reactive arthritis In these seronegative inflammatory conditions an intestinal or urethral infection (usually in an HLA-B27 positive subject) can lead to an arthritis, suggesting a primary role for the mucosal immune system in the pathogenesis of joint inflammation. Patients with Reiter's syndrome have high concentrations of serum $\operatorname{IgA},{ }^{47}$ but this seems to be a common finding in all forms of inflammatory arthritis. Granfors and Toivanen have presented more convincing evidence of an abnormal immune response in patients with reactive arthritis following yersinia infection. ${ }^{48}$ Compared with patients with uncomplicated yersinia infection, those with reactive arthritis have high concentrations of serum IgA antibody to this organism; furthermore, the IgA contains secretory component and is predominantly $\operatorname{IgA} 2$, suggesting an intestinal origin. Eastmond et al, ${ }^{49}$ however, found no significant $\operatorname{IgA}$ response in patients with reactive arthritis following salmonella infection.

It seems that little new light will be shed on the alterations in intestinal immunity in inflammatory arthritis by serum studies. As seen above in our study of intestinal humoral immunity in ankylosing spondylitis, serum antibody response does not always reflect mucosal immunity. Investigators must turn to direct investigation of the intestine if the many questions are to be answered.

\section{Intestinal epithelial barrier, mucosal} permeability, prostaglandins, and NSAIDs The small intestine acts as a selective barrier to material in the gut lumen, allowing absorption of essential nutrients and excluding the rest. If the intestinal mucosa is 'leaky' then potentially antigenic material could gain access to the intestinal lamina propria and the systemic circulation. Possibly, an immune response is thus generated, which may lead to diseases such as inflammatory arthritis. The hypothesis that abnormal intestinal permeability is a primary event in the pathogenesis of inflammatory arthritis has proved attractive and has stimulated much research.

MEASUREMENT OF INTESTINAL PERMEABILITY Intestinal absorption takes place through both active and passive transport mechanisms; passive absorption takes place through transcellular or paracellular routes. In general, small molecules (such as water) are freely permeable and large molecules (such as dextran) have a low rate of permeability. Medium sized molecules have an intermediate rate of mucosal penetration, and it is these substances which are generally 
used as probe molecules for the measurement of intestinal permeability. There is an inverse correlation between molecular diameter and permeability. Several factors govern the selection of probe molecules to demonstrate changes in intestinal permeability. Molecules soluble in lipids are less suitable than molecules absorbed by passive diffusion. Probe molecules must be non-toxic, not metabolised after absorption, and urinary or plasma concentrations must be easily assayed. Three classes of probe molecules have been used: polyethylene glycols, sugars, and radiolabelled ${ }^{51} \mathrm{Cr}$-EDTA. Details of these methods are beyond the scope of this review and have been well reviewed elsewhere. ${ }^{5}$ so

\section{INTESTINAL PERMEABILITY IN RHEUMATIC}

DISEASES

Research in this area has been bedevilled by the fact that NSAIDs alter intestinal permeability, and as many patients with inflammatory arthritides are receiving long term treatment with these agents it is difficult to determine whether reported changes in intestinal permeability are a primary phenomenon in some rheumatic diseases or merely a secondary effect of NSAIDs.

Rheumatoid arthritis Bjarnason et al reported increased intestinal permeability in rheumatoid arthritis using the ${ }^{51} \mathrm{Cr}$-EDTA test ${ }^{6}$; unfortunately, it was not possible to determine whether this was due to NSAID treatment or not. Studies using differential sugar absorption tests have been beset by similar difficulties. In general, it is felt that the observed increase in intestinal permeability is almost certainly due to NSAIDs. We used the cellobiose/mannitol test as a measure of intestinal permeability in a group of 18 patients with seropositive rheumatoid arthritis; six patients were not using NSAIDs, and intestinal permeability was normal in all six. About half of the patients taking NSAIDs had abnormal urinary cellobiose/mannitol ratios (unpublished data).

Ankylosing spondylitis Smith et al $l^{11}$ found increased polyethylene glycol 400 permeability in a group of patients with ankylosing spondylitis, which was independent of NSAID use. With the cellobiose/mannitol test, we showed increased permeability in two patients not taking NSAIDs (unpublished observations). Altered intestinal permeability in ankylosing spondylitis may reflect the subclinical ileal inflammatory changes which have been reported in this condition. Indeed, small intestinal Crohn's disease commonly causes increased intestinal permeability. ${ }^{52}$

In summary, therefore, it seems that altered intestinal permeability in rheumatoid arthritis is secondary to NSAID intake, whereas there is convincing evidence that patients with ankylosing spondylitis have altered permeability de novo.

NSAIDS AND THE SMALL INTESTINE

In clinical practice the stomach and duodenum are the most important sites of NSAID induced side effects, ${ }^{53}$ yet in animal studies it is the small intestine which bears the brunt of NSAID induced damage. ${ }^{54}$

In humans NSAIDs have been reported to cause small intestinal ulceration, ${ }^{55}$ inflammation, ${ }^{7}$ bleeding and perforation, ${ }^{56}$ and stricture formation. ${ }^{57}$ Inflammatory bowel disease may be exacerbated or precipitated by these agents, ${ }^{58}$ and steatorrhoea has been associated with the use of mefenamic acid. ${ }^{59}$

Animal models provide some insight into the mechanisms behind NSAID induced damage of the small intestine. A single large dose of indomethacin, given either orally or subcutaneously, causes multiple small intestinal ulcers within 48 hours. ${ }^{54}$ Ulcers do not occur in germ free animals, ${ }^{60}$ and pretreatment with antibiotics reduces the severity of the damage to the small intestine. ${ }^{61} \mathrm{~A}$ reduction in mucosal prostaglandin synthesis may play a central part in the cause of these lesions as the NSAID induced damage is markedly reduced if prostaglandins are given concurrently. ${ }^{62}$ Del Soldado et al used bigger doses of indomethacin to induce ulcers of the small intestine one to two hours after administration of indomethacin; both prostaglandin $E_{2}$ and cysteamine (a free radical scavenger) were effective in preventing the erosions, but antibiotics had no beneficial effect. This suggests that reduced mucosal prostaglandins and free radicals are important in the early phase of damage to the small intestine, with the later phase being a result of bacterial overgrowth.

Bjarnason and colleagues have defined the effects of NSAIDs on the human small intestine. They used ${ }^{51} \mathrm{Cr}$-EDTA as a probe molecule to show increased intestinal permeability in patients taking these agents ${ }^{6}$; this was noted regardless of the type of arthritis for which these agents were taken. They showed that the increase in intestinal permeability was in proportion to the degree of inhibition by various NSAIDs of mucosal cyclooxygenase. ${ }^{64}$ Further evidence for the central role of decreased cyclooxygenase, and thus reduced mucosal prostaglandin synthesis, is provided by the fact that concurrent prostaglandin administration (as the synthetic derivative misoprostol) prevents NSAID induced changes in small intestinal permeability. ${ }^{65}$ These findings were confirmed by Krugliak et al, who showed that administration of exogenous prostaglandin $E_{2}$ prevented the increase in permeability of polyethylene glycol 400 due to NSAIDs. ${ }^{66}$ Thus prostaglandins have an important cytoprotective role, not only in the stomach and duodenum, but also in the small intestine.

Unfortunately, the mechanism of NSAID induced small intestinal damage may not be due to inhibition of mucosal prostaglandin synthesis alone. Rainsford $e t a l^{67}$ have proposed (from data from animal studies) that the side effects of NSAIDs on the small intestine may be due to the effects of these agents on the enzymes in the Embden-Meyerhof pathway and the tricarboxylic acid cycle, leading to a reduction in intracellular ADP concentrations. Bjarnason et al provided some evidence in support of this hypothesis when they showed that concurrent 
administration of glucose and citrate with indomethacin prevented alterations in small intestinal permeability. ${ }^{68}$ Clearly, the mechanisms whereby NSAIDs alter small intestinal permeability merit further study.

\section{NSAID INDUCED SMALL INTESTINAI INFLAMMATION}

Radiolabelled leucocyte scans have been extensively evaluated as a means of assessing disease extent and activity in patients with inflammatory bowel disease. ${ }^{69}$ Abdominal scintigrams are obtained at four hours and 20 hours after injection of the radiolabelled white cells for localisation of the inflammation, and a four day faecal collection is made for indium-111 estimation, thus providing an objective index of the degree of inflammation. The technique is particularly useful in establishing the presence of ileocaecal inflammation. With the ${ }^{111}$ In leucocyte technique, Bjarnason et al demonstrated inflammation of the small intestine in two thirds of patients (with rheumatoid arthritis or osteoarthritis) taking NSAIDs. ${ }^{7}$ Intestinal inflammation is not evident until patients have taken these agents for at least six months, and the changes may persist for up to 18 months after their discontinuation. Significantly, leucocyte scans were normal in newly diagnosed patients with rheumatoid arthritis-that is, patients not taking NSAIDs; this suggests that inflammation of the small intestine is a manifestation of NSAID use rather than of the inflammatory arthritis itself.

Is NSAID induced inflammation of the small intestine of any clinical significance? Using whole body retention of ${ }^{75} \mathrm{SeHCAT}$ and ${ }^{58} \mathrm{Co}$ labelled vitamin B-12 as a marker of ileal dysfunction, Bjarnason et al showed the presence of significant dysfunction in patients taking NSAIDs, but this was not as severe as in Crohn's disease. ${ }^{7}$ The same group also showed increased intestinal protein loss (using ${ }^{51} \mathrm{Cr}$ labelled proteins) in patients with NSAID induced inflammation. ${ }^{70}$ The protein loss was, however, mild, and only one of nine patients studied had hypoalbuminaemia. Inflammation of the small intestine also seems to be associated with blood loss, as shown by the same group using ${ }^{99 m}$ Tc labelled red blood cells. The radiolabelled red cells accumulated in the same region as the radiolabelled white cells, suggesting that the inflammation leads to blood loss. The authors suggest that this blood loss associated with inflammation of the small intestine might account for unexplained iron deficiency anaemia commonly seen in arthritic patients.

The same group from Northwick Park have described an unusual type of small intestinal stricture in patients taking NSAIDs ${ }^{57}$; these strictures are diaphragmatic, with the lumen narrowed to a pin hole. The strictures may be multiple, and in a series of four patients reported by Bjarnason et al three required resection.

The work on NSAID induced enteropathy reported by the Northwick Park group has stimulated much interest; however, the clinical significance of inflammation of the small intes- tine remains to be established and the work validated by further studies.

Future research in this area will need to focus on intestinal mechanisms, rather than extrapolating data based on studies of blood and peripheral lymphocytes. Advances in this rapidly developing field of research are likely to provide insight not only into the pathophysiology of rheumatic diseases, but may also contribute indirectly to knowledge and treatment of inflammatory bowel disease. ${ }^{71}$

1 Gravallese E M, Kantrowitz F G. Arthritic manifestations of inflammatory bowel disease. Am $\mathcal{f}$ Gastroenterol 1988; 83. 703-9.

2 Wands J R, La Mont J T, Mann E, Isselbacher K J. Arthritis associated with intestinal-bypass procedure for morbid obesity. N Engl f Med 1976; 294: 121-4.

3 Mielants H, Veys E M, Cuvelier C, De Vos M. Ileocolonoscopic findings in seronegative spondyloarthropathies. $\mathrm{Br} \mathcal{J}$ Rheumatol 1988; 27 (suppl 11): 95-105.

4 Ebringer A. The relationship between klebsiella infection and ankylosing spondylitis. Baillieres Clin Rheumatol 1989; 3: 321-38.

5 Katz K D, Hollander D. Intestinal mucosal permeability and rheumatological diseases. Baillieres Clin Rheumatol 1989; 3: 271-84.

6 Bjarnason I, Williams P, So A, et al. Intestinal permeability and inflammation in rheumatoid arthritis; effects of nonsteroidal anti-inflammatory drugs. Lancet 1984; ii: $1171-4$.

7 Bjarnason I, Zanelli G, Smith T, et al. Non-steroidal antiinflammatory drug induced intestinal inflammation in humans. Gastroenterology 1987; 93: 480-9.

8 Svartz N. The treatment of rheumatoid poly-arthritis with acid AZO compounds. Rheumatism 1948; 4: 56-60.

9 Brandtzaeg P, Baklien K. Immunohistochemical studies of the formation and epithelial transport of immunoglobulins in normal and diseased human intestinal mucosa. Scand $\mathcal{F}$ Gastroenterol 1976; 11 (suppl 36): 1-45.

10 Brandtzaeg P, Korsrud F R. Significance of different J chain profiles in human tissues: generation of IgA and IgM with inding site for secretory comp the population, including IgG and IgD producing cells, and depends on the clinical

11 Mestecky J, McGhee J R. Immunoglobulin A (IgA): molecular and cellular interactions involved in IgA biosynthesis and immune response. Adv Immunol 1987; 40: 153-245.

12 Tomasi T B. Structure and function of mucosal antibodies. Annual Review of Medicine 1970; 21: 281-98.

13 Brandtzaeg $P$. The secretory immune system of lactating mammary glands compared with other exocrine organs. Ann NY Acad Sci 1983; 409: 353-81.

14 Freter R. Mechanisms of action of intestinal antibody in experimental cholera. II-Antibody-mediated antibacterial reaction at the mucosal surface. Infect Immun 1970; 2: 556-62.

15 Ogra P L, Karzon D T. Distributions of poliovirus antibody in serum, nasopharynx and alimentary tract following in serum, nasopharynx and alimentary tract following segmental immunisation of lower alimentary

16 Colten $\mathrm{H}$, Bienenstock J. Lack of $\mathrm{C} 3$ activation through classical or alternate pathways by human secretory IgA anti-blood-group-A antibody. In: Mestecky J, Lawton A R eds. The immunoglobulin $A$ system. Vol 45. New York: Plenum, 1974: 305-15.

17 Brandtzaeg P, Baklien K, Bjerke K, Rognum T O, Scott H, Valnes $K$. Nature and properties of the human gastrointestinal immune system. In: Miller $\mathrm{K}$, Nicklin S, eds. Immunology of the gastrointestinal tract. Florida: Boca Raton, 1987: 1-85.

18 Bjarnason I, Peters T J. Helping the mucosa make sense of macromolecules. Gut 1987; 28: 1057-61.

19 Mestecky J, McGhee J R, Arnold R R, Michalek S M, Prince $\mathrm{S} \mathrm{J}$, Babb J L. Selective induction of an immune response $S \mathrm{~J}$, Babb $\mathrm{J} \mathrm{L}$. Selective induction of an immune response in human external secretions by ingest

20 Seidman $\mathbf{E}$, Walker $W$ A. Intestinal defences. In: Kirsner J B, Shorter R G, eds. Inflammatory bowel disease. Philadelphia: Lea and Febiger, 1988: 65-74.

21 Wolf $\mathrm{J}$ L. The membranous epithelial $(M)$ cell: a portal of antigen entry. In: Kirsner J B, Shorter $\mathbf{R}$ G, eds. Inflammatory bowel disease. Philadelphia: Lea and Febiger, 1988: 75-88.

22 Elson $\mathrm{C} \mathrm{O}$. Induction and control of the gastrointestinal immune system. Scand f Gastroenterol 1985; 20 (suppl 114) $1-15$.

23 Mestecky J, McGhee J R, Michalek S M, Arnold R R, Crago $S$ S, Babb J L. Concept of the local and common mucosal immune response. Adv Exp Med Biol 1978; 107: 185-92.

24 Jertborn M, Svennerholm A-M, Holmgren J. Saliva, breast milk, and serum antibody responses as indirect measures of intestinal immunity after oral cholera vaccination or natural disease. F Clin Microbiol 1986; 24: 203-9.

25 Sartor R B. Importance of intestinal mucosal immunity and luminal bacterial cell wall polymers in the aetiology of 
inflammatory joint diseases. Baillieres Clin Rhewmatol 1989; 3: $223-45$

26 Lichtman S N, Sartor R B, Schwab J H. Antibody response to bacterial cell wall fragments in small bowel bacterial overgrowth in rats [Abstract]. Gastroenterology 1988; 94: 262.

27 Sartor R B, Cleland D R, Catalano C J, Schwab J H. Serum antibody to bacterial cell wall peptidoglycan in inflammatory bowel disease patients. Gastroenterology 1985; 88: 1571 .

28 Park H, Schumacher H R, Zeiger A R, Rosenbaum J T. Antibodies to peptidoglycan in patients with spondylarthropathies: a clu

29 Brandtzaeg P, Baklien K. Immunoglobulin producing cells in the intestine in health and disease. Clinical Gastroenterology 1976; 5: 251-69.

30 Brandtzaeg P, Halstensen T S, Kett K, et al. Immunobiology and immunopathology of human gut mucosa: humoral immunity and intraepithelial lymphocytes. Gastroenterolog 1989; 97 : 1562-84.

31 Stodell M A, Butler R C, Zemelman V A, Henry $K$, Brewerton D A. Increased numbers of IgG-containing cells in rectal lamina propria of patients with ankylosing in rectal lamina propria of patients with

32 Segal A W, Isenberg D A, Hariirousou V, Tolfree S, Clark J, Snaith M L. Preliminary evidence for gut involvement in the pathogenesis of rheumatoid arthritis? $\mathrm{Br} \mathcal{F}$ Rheumatol 1986; 25: 162-6.

33 Rooney P J, Jenkins R T, Smith K M, Coates G. "'Indiumlabelled polymorphonuclear leucocyte scans in rheumatoid arthritis - an important clinical cause of false positive results. Br $\mathcal{C}$ Rheumatol 1986; 25: 167-70.

34 Bjarnason I, Hopkinson N, Zanelli G, et al. Treatment of non-steroidal anti-inflammatory drug induced enteropathy. Gun-steroidal anti-inflim

35 Franssen M J A M, Van De Putte L B A, Gribnau F W J. IgA serum levels and disease activity in ankylosing spondylitis: serum levels and disease activity in ankylosing spondylitis:

36 Sanders K M, Hertzman A, Escobar M R, Littman B H Correlation of immunoglobulin and $C$ reactive protein levels in ankylosing spondylitis and rheumatoid arthritis. Ann Rherm Dis 1987; 46: 273-6.

37 Ebringer $R$ W, Cawdell D R, Cowling $P$, Ebringer A. Sequential studies in ankylosing spondylitis: association of Klebsiella pneumoniae with active disease. Ann Rheum Dis 1978; 37: 146-51.

38 Trull A, Ebringer R, Panayi G S, Colthorpe D, James D C O Ebringer A. IgA antibodies in ankylosing spondylitis. Scand I Rhewomatol 1983; 12: 249-53.

39 Trull A K, Ebringer A, Panayi G S, Ebringer R, James D C O. HLA-B27 and the immune response to enterobacterial antigens in ankylosing spondylitis. Clin Exp Immunol 1984; 55: 74-80.

40 Van Bohemen C G, Nabbe A J J $M$, Goie The $H$ S, Mazurkiewicz E S, Van Der Linden S, Zanen H C. Serum IgA antibodies to enterobacteria in a

41 Pease C T, Fordham J N, Stubbs M M, Archer J R. IgA antibodies to gram-negative bacteria in the serum and saliva of patients with ankylosing spondylitis. Scand $\mathcal{F}$ Rheumato 1987; 16: 199-203.

42 Cooper R, Fraser S M, Sturrock R D, Gemmell C G. Raised titres of anti-klebsiella IgA in ankylosing spondylitis, rheumatoid arthritis, and inflammatory bowel disease. BM7 1988; 296: 1432-4.

43 O'Mahony S, Nuki G, Ferguson A. Systemic and intestinal humoral immunity in ankylosing spondylitis: evidence humoral immunity in ankylosing spondylitis: evidence (suppl 2): 92 .

44 Westedt M-L, Daha M R, Baldwin W M, Stijnen T, Cats A Serum immune complexes containing IgA appear to predict erosive arthritis in longitudinal study in rheumatoid arthritis. Ann Rhewn Dis 1986; 45: 809-15.

45 Schroehenloher R E, Koopman W J, Alarcon G S. Molecula forms of IgA rheumatoid factor in serum and synovial fluid of patients with rheumatoid arthritis. Arthritis Rheum 1986 29: 1194-202.

46 MacDermott R P, Nash G S, Bertovich M J, et al. Altered patterns of secretion of monomeric IgA subclass 1 by intestinal mononuclear cells in inflammatory bowel disease. Gastroenterology 1986; 91: 379-85.

47 Inman R D, Johnston E A, Klein M H. Analysis of serum and synovial fluid IgA in Reiter's syndrome and reactive arthritis. Clin Immonol Immumopathol 1987; 43: 195-203. 48 Granfors $\mathrm{K}$, Toivanen A. IgA-anti-yersinia antibodies in yersinia triggered reactive arthritis. Ann Rheum Dis 1986; 45: $561-5$.

49 Eastmond $\mathrm{C}$ J. Gram-negative bacteria and B27 disease. $\mathrm{Br} \mathcal{F}$ Rhermatol 1983; 22: 67-74.

50 Hamilton I. Small intestinal mucosal permeability. In: Pounder R E, ed. Recent advances in gastroenterology. Vol 6. Pounder R E, ed. Recent advances in gastroenter

51 Smith M D, Gibson R A, Brooks P M. Abnormal bowel permeability in ankylosing spondylitis and rheumatoid arthritis. $\mathcal{F}$ Rheumatol $1985,12.299-305$.

52 Ukabam S O, Clamp J R, Cooper B T. Abnormal small intestinal permeability to sugars in patients with Crohn's disease of the terminal ileum and colon. Digestion 1983; 27: $70-4$.

53 Hawkey C J. Non-steroidal anti-inflammatory drugs and peptic ulcers. BMF 1990; 300: 278-84.

54 Kent T H, Cardeli R M, Stanler F U. Small intestinal ulcers and intestinal flora in rats given indomethacin. $A m \mathcal{F}$ Pathol 1969; 54: 237-45.

55 Madhok R, Mackenzie J A, Lee F D, Bruckner F E, Terry T R, Sturrock R D. Small intestinal ulceration in patients receiving non-steroidal anti-inflammatory drugs for rheumatoid arthritis. $Q \mathcal{F}$ Med 1986; 255: 53-8.

56 Langman M J, Morgan L, Worrall A. Use of anti-inflammatory drugs by patients admitted with small and large bowel perforations and haemorrhage. $B M \mathcal{F} 1985 ; 290$ : 347-9.

57 Bjarnason I, Price A B, Zanelli G, et al. Clinicopathological features of nonsteroidal anti-inflammatory drug-induced small intestinal strictures. Gastroenterology 1988; 94: $1070-4$.

58 Kaufmann H J, Taubin H L. Nonsteroidal anti-inflammatory drugs activate quiescent inflammatory bowel disease. Ann Interm Med 1987; 107: 513-6.

59 Marks J S, Gleeson M H. Steatorrhoea complicating therapy with mefenamic acid. BMF 1975; iv: 442

60 Robert A, Asano T. Resistance of germ-free rats to indomethacin-induced intestinal lesions. Prostaglandins 1977; 14: $331-41$.

61 Satoh H, Guth P H, Grossman M I. Role of bacteria in gastric ulceration produced by indomethacin in the rat: cytoprotective action of antibiotics. Gastroenterology 1983; 84: 483-9.

62 Robert A. Cytoprotection by prostaglandins. Gastroenterology 1975; 77: 761-7.

63 Del Soldado P, Foschi D, Benoni G, Velo G P. Early and late phases in the formation by anti-inflammatory drugs of intestinal lesions in rats. In: Rainsford K D, Velo G P, eds. Side effects of anti-inflammatory drugs. Lancaster: MTP Press, 1987: 67-81.

64 Bjarnason I, Williams P, Smethurst P, Peters T J, Levi A The effect of non-steroidal anti-inflammatory drugs and The effect of non-steroidal anti-inflammatory drugs and prostaglandins on the perme
bowel. Gut 1986; 27: 1292-7.

65 Bjarnason I, Smethurst P, Fenn C G, Lee C E, Menzies I S Levi A J. Misoprostol reduces indomethacin induced changes in human small intestinal permeability. Dig Dis Sci 1989; 34: 407-11.

66 Krugliak P, Hollander D, Le K, Ma T, Dadufalza V D, Katz $K$ D. Regulation of polyethylene glycol 400 intestinal permeability by endogenous and exogenous prostanoids. Influence of non-steroidal anti-inflammatory drugs. $G u$ 1990; 31: 417-21.

67 Rainsford K D. Prevention of indomethacin-induced gastrointestinal ulceration in rats by glucose-citrate formulations: role of ATP in mucosal defences. Br $¥$ Rheumatol 1987; 26 role of ATP (suppl 2): 81 .

68 Bjarnason I. Non-steroidal anti-inflammatory drug-induced small intestinal inflammation in man. In: Pounder R E, ed. Recent adoances in gastroenterology. Vol 7. London Churchill Livingstone, 1988: 23-46.

69 Saverymuttu S H, Peters A M, Lavender J P, Hodgson H J, Chadwick V S. 'II'Indium autologous leucocytes in inflammatory bowel disease. Gut 1983; 24: 293-9.

70 Bjarnason I, Zanelli G, Prouse P, et al. Blood and protein loss via small intestinal inflammation induced by non-steroidal anti-inflammatory drugs. Lancet 1987; ii: $711-4$.

71 Bjarnason I, Peters T J. Intestinal permeability, non-steroidal anti-inflammatory drug enteropathy and inflammatory bowel disease: an overview. Gut 1989; 30: 22-8. 